\title{
MODERATE-THROUGHPUT IDENTIFICATION AND COMPARISON OF CAMPYLOBACTER-INFECTING BACTERIOPHAGES
}

Tylor J. Johnson ${ }^{a}$, Janette M. Shank ${ }^{a}$, Kishen M. Patel ${ }^{a}$, Maribel D. Paredes ${ }^{a}$, Esther D.

Lee $^{a}$, Mary K. Mitchell ${ }^{a}$,Thomas G. Denes ${ }^{b}$, Jeremiah G. Johnson ${ }^{\text {a\# }}$

${ }^{a}$ Department of Microbiology, The University of Tennessee, Knoxville, TN 37996, USA

${ }^{b}$ Department of Food Science, The University of Tennessee, Knoxville, TN 37996, USA

\#Corresponding Author:

Jeremiah G. Johnson

M409 Walters Life Sciences

1414 Cumberland Avenue

Knoxville, TN 37996

Ph: (865) 974-6229

E-mail: jjohn358@utk.edu

\section{Running Title}

Identification of Campylobacter bacteriophage

\section{Abstract Word Count: 198}

Text Word Count: 4875 


\author{
Abbreviations \\ CDC: Centers for Disease Control and Prevention \\ CPS: Capsular polysaccharide \\ CS: Campylobacter-specific \\ FDA: Food and Drug Administration \\ GBDP: Genome BLAST Distance Phylogeny \\ GBS: Guillain-Barré Syndrome \\ IBS: Irritable bowel syndrome \\ IC50: Half maximal inhibitory concentration \\ $\mathrm{MH}$ : Mueller-Hinton \\ MIC: Minimum inhibitory concentration \\ ORF: Open reading frame \\ OTU: Operational taxonomic unit \\ PBS: Phosphate-buffered saline \\ PV: Phase variation \\ RA: Reactive arthritis \\ SFCA: Surfactant-free cellulose acetate \\ SM: Saline-magnesium \\ TEM: Transmission electron microscopy \\ VICTOR: Virus Classification and Tree Building Online Resource \\ WHO: World Health Organization \\ WWTP: Wastewater treatment plant
}

\title{
Keywords
}

Anti-infectives; Bacteriophage; Campylobacter; Microbiome; Phylogenomics

\section{Abstract}

Campylobacter jejuni is a leading cause of foodborne infection due to its ability to asymptomatically colonize agricultural animals. In addition to its prevalence, Campylobacter is becoming increasingly resistant to the clinical antibiotics, azithromycin and ciprofloxacin. As a result, public health agencies have identified drug resistant Campylobacter as a serious threat to public health and have suggested combating the pathogen at the farm-level by reducing its burden within agricultural animal reservoirs. 
Due to antibiotic restrictions in agricultural animals, reducing Campylobacter burden on the farm requires the identification and development of novel approaches. To this end, our group employed a broad sampling strategy to isolate a diverse collection of bacteriophages that can predate Campylobacter. We successfully isolated 70 bacteriophages from these samples and subjected them to whole-genome sequencing and initial comparative genomic analysis. Following this analysis, we performed doseresponse assays by adding increasing concentrations of bacteriophage to $C$. jejuni cultures. From this work, we were able to identify diverse phage groups from multiple sources that correlated with robust inhibition of $C$. jejuni growth. Lastly, we employed transmission electron microscopy to examine the morphology of these bacteriophages and were able to confirm that genetic diversity reflected morphological diversity in these bacteriophages.

\section{Importance}

Globally, Campylobacter jejuni is the most common cause of bacterial-derived gastroenteritis due to its ability to colonize agriculturally relevant animals. The increasing prevalence of antibiotic resistant $C$. jejuni strains necessitates the development of novel treatments to combat colonization of the animal host. In this study, we describe the isolation of 70 bacteriophages from various environmental sources that infect and kill C. jejuni. A robust analysis of the genetic diversity of these phage is described, and transmission electron microscopy is utilized to evaluate morphological differences. This study describes a novel prospecting strategy for 
isolating bacteriophage that infect $C$. jejuni from various environmental sources. In previous studies, bacteriophage that kill C. jejuni have been isolated from sewage and pig manure; however, the majority have been isolated from chickens, and have been relatively few in number. We postulate this study increases the likelihood of isolating bacteriophage efficacious at combating C. jejuni colonization of agricultural animal hosts at the farm-level or treating infected humans.

\section{Introduction}

Campylobacter jejuni is a leading cause of bacterial-derived gastroenteritis in both the developed and developing world. In the developed world, the bacterium is thought to most often infect humans following the consumption of undercooked poultry, especially chickens, primarily due to the bacterium's ability to commensally reside within the avian gastrointestinal tract. Following ingestion, Campylobacter adheres to and invades epithelial cells lining the gastrointestinal tract, causing a potent inflammatory response that typically results in moderate to severe diarrhea, fever, and abdominal cramps (1-3). While most infections in the developed world are self-limiting, and patients often recover without treatment, there are several post-infectious disorders that can complicate recovery, including septicemia, Guillain-Barré Syndrome (GBS), irritable bowel syndrome (IBS), and reactive arthritis (RA) (4). In addition to the prevalence and severity of these infections, the extensive use of antibiotics in agriculture and human medicine is responsible for an emergence of antibiotic-resistant Campylobacter (5). Thus, the Centers for Disease Control and Prevention (CDC) has designated antibiotic- 
resistant Campylobacter as a 'serious threat' to public health in the United States (6). Taken together, these observations indicate that the development of novel antimicrobials for the reduction or treatment of human Campylobacter infections is necessary (7).

Several avenues of reducing the incidence of human campylobacteriosis have been pursued at various levels of the infectious cycle (3). As mentioned above, in the developed world, the most common infection source in humans is undercooked poultry. Thus, a large amount of research has centered on preventing contamination of food by reducing the pre-harvest burden of Campylobacter in poultry flocks (8). Some of this research includes the identification and initial development of bacteriocins, bacteriophage, natural products, probiotics, small molecule inhibitors, and vaccines (3). While some of these studies demonstrated modest reductions of $C$. jejuni numbers in small chicken cohorts, none have accomplished widespread implementation at the farm level.

As mentioned above, using bacteriophage at the farm-level to reduce Campylobacter numbers in poultry and decrease transmission to the food chain is an intervention that has been proposed by us and others (8-11). Bacteriophage are infectious agents of bacteria, ubiquitous in the environment where the host bacterium resides, and hold potential to be developed into antimicrobial treatments. Historically, bacteriophages were pursued as treatments for human infections in Europe and Asia (8), while the United States invested heavily in the identification and development of antibiotics. With the emergence of antibiotic-resistant microorganisms, several research groups have begun to re-examine the efficacy of various bacteriophage at treating these 
resistant infections $(8,12-14)$. Additionally, since phages often exhibit specificity for a particular bacterial host, treatment may be accomplished without impacting the normal microbiota, a feature that is becoming more appreciated in animal and human health. As such, it is intriguing whether the earlier work to isolate Campylobacter-specific bacteriophage can be broadened and leveraged to develop treatments that reduce colonization of animal hosts, including chickens (8). There is precedence for phage as a biocontrol treatment being commercially approved in the United States, and several phage-based biocontrol products have been approved for use on food (15).

In previous studies, bacteriophages with anti-Campylobacter activity have been isolated from sewage, pig manure, poultry carcasses, and broiler chickens in Europe (8, $10,11,16-19)$. To increase the breadth and efficiency of anti-Campylobacter phage identification and characterization, we developed a phage prospecting pipeline for use in our studies. In this work, we describe the isolation of bacteriophage from multiple agricultural and environmental sources, including Campylobacter-free commercial chickens, which was confirmed via 16S rRNA gene analysis. Following phage purification, phage genomes were extracted from 70 phage suspensions and sequenced. From these sequencing runs, we were able to produce 58 de novo assemblies and determine the relative diversity of these phages using the Virus Classification and Tree-building Online Resource (VICTOR). Dose-response analyses of the phage suspensions were conducted and we were able to determine which group (determined by VICTOR) of bacteriophage correlated with the greatest inhibition of $C$. jejuni in vitro growth. Finally, transmission electron microscopy (TEM) was used to evaluate the morphology of bacteriophages isolated exhibiting the most potential to kill 
C. jejuni in vitro, leading to the identification of several unique anti-Campylobacter phage morphologies. The establishment of this phage prospecting pipeline will not only allow us to examine the diversity of anti-Campylobacter phage in each environment, but will also aid us in identifying those that hold potential as treatments for reducing Campylobacter colonization of animal hosts, including poultry, and/or human infections.

\section{Results and discussion}

\subsection{Isolation and purification of bacteriophage from environmental samples}

The following phage numbers were isolated and purified from environmental samples (letters in parenthesis annotate what environment phages were isolated from): 51 from chickens from a large-scale processor (A), 2 from small farm chickens (B), 14 from untreated wastewater from a wastewater treatment plant (WWTP) (C), 2 from treated wastewater excluding disinfection from another WWTP (D), and 1 from pig feces (E). Samples potentially containing phage were diluted on $\mathrm{MH}+$ top agar plates to determine if phage were present and at what dilution (Fig. S1A). These phages had various plaque morphologies and zone of clearing sizes, indicating different phage strains (Fig. S1B and S1C). Several other environmental samples yielded plaques, however, bacteriophage could not be consistently propagated from these samples during the purification steps.

\subsection{Campylobacter cell counts}

Campylobacter spp. counts from each sample are shown in Table 1. Fecal samples from cows contained $10^{3}-10^{5} \mathrm{CFU} / \mathrm{g}$ Campylobacter, fecal samples from 
chickens on a small farm contained $10^{6}-10^{7} \mathrm{CFU} / \mathrm{g}$ Campylobacter, and fecal samples from sheep contained $10^{4}-10^{5} \mathrm{CFU} / \mathrm{g}$ Campylobacter. Human wastewater and pig feces did not yield countable Campylobacter colonies due to the presence of fungi in those samples and subsequent fungal growth on the Campylobacter-selective media. There was no Campylobacter detected in any of the 50 chickens obtained from large flocks at a processor on two different occasions. Various studies have reported rates of Campylobacter colonization in chickens from $22-87 \%$ and that this prevalence can be highly variable (20). However, Campylobacter-free farms do exist in the US and the UK $(21,22)$. Elucidating the reason that Campylobacter-infecting bacteriophage are present in chicken ceca that do not contain Campylobacter is of great interest to our research group.

\subsection{Chicken microbiome evaluation via 16s rRNA analysis}

Using a more sensitive method of identifying Campylobacter within the cecal contents of chickens, we characterized the microbiomes of 10 of the cecal contents harvested from the large processor by sequencing and analyzing the 16S rRNA genes present within each sample. Additionally, we analyzed the microbiomes of five chickens inoculated on the day-of-hatch with $10^{6}$ CFU C. jejuni 81-176 (positive control). Another group of five chickens was mock-infected on the day-of-hatch with sterile PBS (negative control). Sequencing data was organized into operational taxonomic units (OTUs) using mothur (23), and the summation of each order, family, or genus was calculated. The results from this are shown in Figure 1 and Table S1 (Supplemental data). As expected, chickens infected on day-of-hatch with $C$. jejuni 81-176 yielded 16S rRNA sequences 
that corresponded to the microorganism when the cecum was harvested 7 days postinfection. The chickens that were mock-infected with PBS had a microbiota very similar to that of the infected birds, but did not contain sufficient Campylobacter sequences at the time of harvest. Similarly, the cecal samples from chickens at the large processor did not have sufficient Campylobacter sequences detected.

As expected, the mock-infected and Campylobacter-infected chickens that were hatched from specific pathogen-free eggs and raised in-house had a much less diverse cecal microbiome when compared to the chickens harvested from the processor that originated from large farms. The cecal microbiomes of these chickens were similar to what has been observed in previous studies, with the predominant bacterial taxa including Firmicutes, Bacteroidetes, and Proteobacteria (24-26). These microbiomes can include a relatively small population of Campylobacter, which were present in the Qu (26) and Sergeant (25) studies, or can be free of Campylobacter, like in this study and the Wei (24) study.

Typically, bacteriophages have specific host ranges. Thus, the isolation of bacteriophages with anti-Campylobacter activity from 49 of the 50 chicken ceca originating from large farms that did not contain Campylobacter was surprising, but not entirely unexpected. In a previous study, using culture-dependent techniques, Campylobacter was not detected in $71 \%$ of bacteriophage-positive chicken ceca. The authors postulated that this observation may have been due to the phage reducing Campylobacter numbers below the limit of detection in the ceca or during experimental recovery (12). Another possibility for this observation is that Campylobacter has 
colonized upstream of the ceca in the gastrointestinal tract and the phage have cleared the bacteria downstream.

Another possible cause of the bacteriophage occurring in chicken ceca that do not harbor $C$. jejuni is that there is an alternate bacterial host. Most studies consider a bacteriophage host range to be broad when it infects multiple strains within a single species. However, measuring host range is dependent on the techniques used (i.e. spot testing, plaquing, etc.) and the growth conditions (e.g., incubation, temperature, and growth media) used for determination (27), making it difficult to compare host range results from different studies. To the best of our knowledge, no bacteriophage with antiCampylobacter activity have been isolated with a host range that extends beyond infection of closely related strains within the same species. Investigating the efficacy of the bacteriophage described in this study against a broad range of Campylobacter and closely related species is a future direction of this work.

\subsection{Dose-response assays to screen bacteriophage for anti-Campylobacter potency}

Ten-fold serial dilutions $\left(1-10^{6}\right)$ of each bacteriophage isolated from this study were screened for their ability to inhibit $C$. jejuni 81-176 growth in a 96-well microplate format. The percent growth of $C$. jejuni 81-176 at the bacteriophage titer (PFU) where growth was inhibited by at least $50 \%$ (IC50) is shown in Figure 2. These assays indicated the phage strains exhibited a broad spectrum of activity against $C$. jejuni 81176. Approximately $22 \%$ of the phages yielded an IC50 of 1 PFU, $22 \%$ exhibited an IC50 of 10 PFU, $22 \%$ had an IC50 between $10^{2}$ and $10^{6}$ PFU, and $32 \%$ did not inhibit Campylobacter growth. 
Bacteriophages were isolated in this study from environmental samples that varied in temperature from ambient (temperature of the environment at the time of collection) to $42^{\circ} \mathrm{C}$ (chicken body temperature). For dose-response analysis, we chose to conduct the assays at $37^{\circ} \mathrm{C}$ for three reasons: i) there was concern that phage isolated from an ambient environment could not be propagated at $42^{\circ} \mathrm{C}$, ii) previous studies have used $37^{\circ} \mathrm{C}$ for bacteriophage propagation $(28,29)$, and iii) a future goal of this work is to develop the phages for treatment of Campylobacter colonization in animals other than chickens, including humans. A future direction of this study is to screen the most promising phages and screen them against a panel of Campylobacter strains at $42^{\circ} \mathrm{C}$ to determine which phages have potential to be developed into a biocontrol treatment for inhibiting Campylobacter colonization in chickens. Another future direction is to determine the phage receptor recognized by these bacteriophages. Typically, bacteriophage that infect $C$. jejuni use capsular polysaccharides (CPS) or flagella as binding receptors (9). Since C. jejuni strains utilize phase variation (PV) to create phenotypic heterogeneity of phage receptors and become resistant to phage (30), it may be necessary to use a cocktail of phages that bind different receptors to maintain reduction of $C$. jejuni colonization in animal hosts.

\subsection{Determination of bacteriophage diversity}

Bacteriophage genetic diversity was visualized using phylogenomic Genome BLAST Distance Phylogeny (GBDP) trees (31) of bacteriophage assemblies that were generated in this study and two previously characterized phages, CP220 and CPt10 (32). The accession numbers for these bacteriophage assemblies are listed in Table 2. 
The OPTSIL clustering program (33) yielded 38 species, 12 genera, and 5 families as defined by VICTOR (Figure 3). The color that phage isolates are highlighted in Figure 3 correspond to their efficacy at killing $C$. jejuni as determined by dose response assays. Hereafter, phage will be described by this color.

Phage distribution based on Clade clustering, as determined by VICTOR (VICTOR refers to Clades as Families), and isolation source of the phage, categorized by the color that indicates their efficacy at killing C. jejuni 81-176, is shown in Figure 4. Categories that had less than 3 phage isolates are not included in the figure. There was one highly potent (indicated as green) and one non-potent (indicated as red) phage clustered in Clade 1, and both phages were isolated from chickens at the large processor. There was one red phage clustered in Clade 2 and it was isolated from a chicken on a small farm. The majority of phages clustered in Clade 3 were green phage $(61.3 \%)$, and 16 of these phages were isolated from the large processor, 1 from the small farm, 12 from untreated wastewater, and 2 from treated, non-disinfected wastewater (Figure 4A). There were more red phages (41.7\%) clustered in Clade 4 than moderately potent (indicated as yellow) or green phage (Figure 4B). Twenty-two of these bacteriophages were isolated from the large processor, 1 from untreated wastewater, and 1 from pig feces. CP220 and CPt10 were clustered into Clade 5.

These results suggest that bacteriophage from Clade 3 have a higher probability of exhibiting robust anti-Campylobacter activity compared to the other Clades and that the phage belonging to this Clade were primarily isolated from large flocks or wastewater. Since a majority of phage clustered into Clade 4 also occurred in large flocks, but were generally not as effective at killing C. jejuni as Clade 3 phage, it will be 
important to distinguish these phages from those in Clade 3 in subsequent prospecting. Clade 1 and Clade 2 only had 3 total bacteriophages isolated, thus more isolates would need to be evaluated to make a stronger conclusion regarding phages from these Clades.

From a source perspective, the majority of phages isolated from untreated wastewater were categorized as highly potent (76.9\%) (Figure $4 \mathrm{C})$. The categories of phage isolated from chickens in large flocks were fairly evenly distributed (27.5 $37.5 \%$ ) (Figure 4D). One yellow phage and one red phage were isolated from small farm chickens; one green phage and one yellow phage were isolated from treated, disinfected wastewater; and one red phage was isolated from pig feces. These results suggest that bacteriophages isolated from untreated wastewater are more likely to exhibit a potent activity against $C$. jejuni compared to phages isolated from the other environments investigated in this study. These results also indicate that bacteriophage diversity is greatest in chickens in large flocks and that some are potent inhibitors of $C$. jejuni growth. This suggests that in further phage prospecting, it will be more efficient to focus on samples from human wastewater and large chicken flocks.

CP220 and CPt10, two previously sequenced Campylobacter bacteriophages (32), were compared to the phage isolated in this study. These two phages are closely related and are members of the class II Campylobacter Myoviridae phages. Of the over 200 bacteriophages isolated in previous studies that have been previously shown to infect C. jejuni or C. coli, the majority belong to the Myoviridae family, although 10 have been isolated that belong to the Siphoviridae family (34). Based on the comparative genomic analysis performed using VICTOR, the phages isolated in this study belong to 
four different Clades, and none are in the same Clade as CP220 and CPt10. Thus, a future direction of this study is to conduct a more robust genomic analysis to further classify these phages, and other previously sequenced bacteriophages that infect Campylobacter, which will allow for a more precise taxonomic classification.

\subsection{Transmission electron microscopy}

Several bacteriophages isolated in this study were observed via TEM (Fig. 5). These images allowed us to correlate the morphology of the phages with the genetic classifications determined by VICTOR. Phage C13 (Fig. 5A) and Phage A126 (Fig. 5B) belong to the same Clade. Phage A136 (Fig. 5C) is in a different Clade than the other phages in Figure 5. As indicated by the clustering and observed in the images, phages C13 and A126 have icosahedral heads, C13 has a flexible tail, and A126 has a rigid tail. A136 has an icosahedral head and flexible tail. The morphological diversity between the imaged phages was reflected in the genomic diversity observed using VICTOR; providing support to the reliability of this online resource. As with the comparative genomic analysis above, a future direction of this study is to ascertain which morphologies (e.g. features) correlate with the greatest inhibition of Campylobacter growth. We postulate the phage prospecting strategy described in this study will streamline future phage identification and development as we will be able to quickly determine which phages, from a genomic and morphological perspective, are likely most efficacious as Campylobacter treatments.

\subsection{Conclusion}


In this study, we describe a pipeline for isolating bacteriophages from various environments that can infect $C$. jejuni and inhibit in vitro growth. The majority of previous studies that isolated $C$. jejuni phages, only isolated from chickens, which may be disadvantageous as a prospecting strategy. We hypothesized that other environments may have a higher probability of containing potent phages that can successfully reduce Campylobacter colonization of animals. We demonstrated that the bacteriophages isolated in this study exhibited a spectrum of activity against $C$. jejuni 81-176 in vitro. Further, we were able to attribute the highest levels of activity to large flocks and human wastewater sources, which contained diverse phages from a genomic and morphological perspective.

A future direction of this work is to study the most effective anti-Campylobacter phages in bioreactor systems that mimic the gastrointestinal environment and animal model systems, including chickens. In order to become a feasible pre-harvest biocontrol treatment, bacteriophage must survive ingestion by the animal, survive enteric defense systems, and bind specifically to the bacterium with minimal non-specific binding to intestinal components. Finally, the bacteriophage must be able to infect Campylobacter and replicate in the gastrointestinal tract (35). Due to the current prevalence of antibiotic resistant $C$. jejuni strains, novel strategies (i.e. phage as a biocontrol treatment) must be developed to decrease the rate of infection from agricultural animals. Additionally, the development of phage therapies may be attractive alternatives for treating Campylobacter infection in patients in the developing world since they are relatively cheap to produce, spare the commensal microbiota (particularly important for malnourished and immunocompromised patients), and can be dry powder formulated so 
that no refrigeration is necessary (36). The ability to eliminate Campylobacter in a specific manner is particularly important in developing nations since the gastrointestinal dysbiosis caused by traditional antibiotics puts patients at a further disadvantage for digestion and nutrient acquisition $(3,37)$. We postulate bacteriophages that infect and kill Campylobacter have the potential to be developed into treatments, but further development and investigation is necessary.

\section{Materials and methods}

\subsection{Microbial strains, maintenance, and culture conditions}

C. jejuni 81-176 has been described in previous studies $(38,39)$. For long term storage, the strain was stored at $-80^{\circ} \mathrm{C}$ in Mueller-Hinton $(\mathrm{MH})$ broth and $20 \%$ glycerol. Inoculum for the experiments described below was grown on $\mathrm{MH}$ agar with $10 \mu \mathrm{g} / \mathrm{mL}$ trimethoprim and incubated for 48 hours at $37^{\circ} \mathrm{C}$ in microaerobic conditions $\left(85 \% \mathrm{~N}_{2}\right.$, $10 \% \mathrm{CO}_{2}, 5 \% \mathrm{O}_{2}$ ). To suspend the bacteria in liquid media, $1 \mathrm{~mL}$ of $\mathrm{MH}$ broth was added to the agar plates containing C. jejuni and a sterile inoculating loop was used to thoroughly mix the inoculum. This inoculum was then transferred to sterile $15 \mathrm{~mL}$ conical tubes containing $14 \mathrm{~mL} \mathrm{MH}$ broth for use in the experiments described below.

\subsection{Isolation and purification of bacteriophage from environmental samples}

To isolate bacteriophage from the environment, liquid samples were collected from two human wastewater facilities. From one facility, samples were collected from the effluent channel of the aerated grit chambers. The samples from this facility represented untreated wastewater. Samples from the other facility were collected from 
the effluent channel just after flowing over the effluent weirs. The samples from this facility represented completely treated wastewater excluding disinfection. Feces were collected from pigs, cattle, and sheep. Chicken cecal contents from a large flock and a small organic farm were also collected.

To prepare solid media for determining the presence of Campylobacter bacteriophage, C. jejuni 81-176 was harvested as described in Section 3.1. These suspensions were diluted to a final $\mathrm{OD}_{600}$ value of 1.0 . Next, $100 \mu \mathrm{L}$ of this culture was transferred to $5 \mathrm{~mL}$ liquid $\mathrm{MH}$ + salts top overlay agar $(10 \mathrm{mM} \mathrm{MgSO}$, $1 \mathrm{mM} \mathrm{CaCl}$, $0.7 \%$ agar). This mixture was poured on top of an $\mathrm{MH}$ agar plate and allowed to solidify at room temperature. To determine if Campylobacter bacteriophage were present in the samples, $1 \mathrm{~g}$ or $1 \mathrm{~mL}$ of the sample was diluted in $9 \mathrm{~mL}$ saline-magnesium (SM) buffer (50 mM Tris- $\mathrm{HCl}, \mathrm{pH} 7.5,0.1 \mathrm{M} \mathrm{NaCl}, 8 \mathrm{mM} \mathrm{MgSO}_{4} \cdot 7 \mathrm{H}_{2} \mathrm{O}, 0.01 \%$ gelatin) and vortexed for 2 minutes. The samples were then centrifuged at 9400 rcf for 1 minute, and $1 \mathrm{~mL}$ of the diluted samples were filtered through a $0.22 \mu \mathrm{M}$ surfactant-free cellulose acetate (SFCA) syringe filter into a $1.5 \mathrm{~mL}$ microcentrifuge tube. For long term storage, both the unfiltered and filtered samples were stored at $4^{\circ} \mathrm{C}$. To determine if phage were present in each sample, an enrichment step was necessary to yield sufficient titers for subsequent steps. Briefly, a $100 \mu \mathrm{L}$ aliquot of filtered sample was transferred to a microcentrifuge tube containing $1 \mathrm{~mL}$ of $C$. jejuni culture, prepared as described in Section 2.1. The cultures were then incubated statically for 24 hours at $37^{\circ} \mathrm{C}$ in microaerobic conditions $\left(85 \% \mathrm{~N}_{2}, 10 \% \mathrm{CO}_{2}, 5 \% \mathrm{O}_{2}\right)$.

After incubation, the cultures were filter sterilized as described above to remove C. jejuni cells and $100 \mu \mathrm{L}$ aliquots from this sample were serially diluted in $900 \mu \mathrm{L}$ SM 
buffer $\left(10^{-2}-10^{-8}\right.$ dilutions $)$ in 96 -deep well plates. Ten $\mu \mathrm{L}$ aliquots of each dilution were spotted onto a $\mathrm{MH}$ top agar plate embedded with $C$. jejuni 81-176 (described above) and plates were incubated for 24 hours at $37^{\circ} \mathrm{C}$ in microaerobic conditions $\left(85 \% \mathrm{~N}_{2}\right.$, $10 \% \mathrm{CO}_{2}, 5 \% \mathrm{O}_{2}$ ). After 24 hours of incubation, the plates were examined for the presence of zones of clearing, indicating bacterial lysis from phage. The dilution yielding zones of clearing was then embedded in an agar embedded C. jejuni culture and incubated for 24 hours at $37^{\circ} \mathrm{C}$ in microaerobic conditions. After 24 hours, individual plaques could be observed in the agar embedded $C$. jejuni culture. A blunt-end pipet was used to excise the agar plug containing the plaque and was transferred to a $1.5 \mathrm{~mL}$ microcentrifuge tube containing $1 \mathrm{~mL}$ SM buffer; this was incubated at $4^{\circ} \mathrm{C}$ on a gyratory platform shaker at $15 \mathrm{rpm}$ for $\sim 24 \mathrm{~h}$. To ensure that samples contained individual phage strains and not a mixture, two subsequent propagation and plaque isolation steps were performed as described above. After the third plaque isolation and purification, the purified phage isolate was enriched in $\mathrm{MH}$ broth with $C$. jejuni 81-176 as described above and phage titers were determined. If there was greater than $10^{7} \mathrm{PFU} / \mathrm{mL}$, the concentrated phage isolate was stored long-term in SM buffer at $4^{\circ} \mathrm{C}$ as described above.

\subsection{Campylobacter cell counts}

To enumerate Campylobacter from the agricultural and environmental samples that were evaluated for phage, samples were serially diluted (1:10) in sterile phosphatebuffered saline (PBS). Dilutions $(100 \mu \mathrm{L})$ were plated on Campylobacter-specific (CS) media (MH agar with 10\% defibrinated sheep blood containing $100 \mu \mathrm{g} / \mathrm{mL}$ 
cyclohexamide, $100 \mu \mathrm{g} / \mathrm{mL}$ vancomycin, $40 \mu \mathrm{g} / \mathrm{mL}$ cefoperazone, and $10 \mu \mathrm{g} / \mathrm{mL}$ trimethoprim) and incubated until countable colonies were present ( $\sim 8$ hours) in microaerobic conditions $\left(85 \% \mathrm{~N}_{2}, 10 \% \mathrm{CO}_{2}, 5 \% \mathrm{O}_{2}\right)$.

\subsection{Chicken microbiome evaluation via 16S rRNA analysis}

To evaluate the cecal microbiome of chickens harvested from large flocks at a processor, a randomized block design was used. Using the random number generator function of the Simetar add-in for Microsoft Excel (40), 10 chickens were chosen from each of two collection dates at the processor (the chickens from the first collection date originated from one farm, and the chickens from the second collection date originated from another farm). Cecal microbiomes of five chickens infected with $C$. jejuni (positive control) and five chickens mock infected with PBS (negative control) were also evaluated.

To infect chickens for microbiome analysis, the day-of-hatch chicken model was used, as previously described, with modifications (41). To prepare the inoculum, $C$. jejuni was cultivated and transferred to a $15-\mathrm{mL}$ conical as described in Section 2.1, but PBS rather than $\mathrm{MH}$ broth was used as a diluent. From this, a suspension of $10^{7}$ CFU/mL C. jejuni 81-176 was made in sterile PBS and used to inoculate day-of-hatch white leghorn chicks (Charles River, Wilmington, MA, USA) by oral gavage with $10^{6}$ CFU C. jejuni (100 $\mu \mathrm{L}$ of suspension). Cecal contents were harvested seven days' postinfection, serially diluted (1:10) in PBS, and plated to enumerate $C$. jejuni. The remaining cecal contents were stored at $-80^{\circ} \mathrm{C}$. This chicken protocol was reviewed and 
approved by the University of Tennessee Institutional Animal Care and Use Committee (protocol \#2493).

To extract bacterial DNA from the stored cecal contents, the DNeasy PowerSoil Kit was used (MO BIO Laboratories, Carlsbad, CA, USA). For 16S rRNA gene sequencing, Illumina's 16s metagenomic sequencing library protocol was utilized per the manufacturer's instructions (42). The V3 - V4 hypervariable region of the 16S rRNA gene was sequenced using the Illumina MiSeq platform and the data was analyzed with the bioinformatics software, mothur, using the MiSeq SOP (23). The cutoff point for minimum representation of genus, family, or order was 10 sequences. Thus, any microorganisms with $\leq 10$ sequences detected were omitted from the dataset.

\subsection{Dose-response assays to screen bacteriophage for anti-Campylobacter potency}

The efficacy of each bacteriophage isolate at inhibiting C. jejuni growth was evaluated via dose-response assays. First, phage stocks were each normalized to a specific titer prior to dilution. Next, 10-fold serial dilutions $\left(1-10^{6}\right)$ of bacteriophage were combined in $\mathrm{MH}$ broth with $C$. jejuni $81-176\left(\mathrm{OD}_{600} 0.025\right)$ in a 96-well microplate and incubated at $37^{\circ} \mathrm{C}$ in microaerobic conditions $\left(85 \% \mathrm{~N}_{2}, 10 \% \mathrm{CO}_{2}, 5 \% \mathrm{O}_{2}\right)$. C. jejuni growth was monitored daily via absorbance $\left(O D_{600}\right)$ in a microplate reader. These trials concluded once bacterial growth reached stationary phase ( 70 h). IC50 values were determined for each phage isolate at daily time points using regression analysis in GraphPad Prism 7.

\subsection{Determination of bacteriophage diversity}


To obtain enough phage DNA to sequence, phages were propagated as described above, enriched using C. jejuni 81-176. Prior to phage capsid lysis, bacterial DNA was treated with 20 U RNAse-free DNase I (Ambion, Inc., Austin, TX, USA), incubated for $15 \mathrm{~min}$ at room temperature, and inactivated at $75^{\circ} \mathrm{C}$ for $5 \mathrm{~min}$, to allow for specific enrichment of phage DNA. Bacteriophage DNA was then isolated and purified using a phage DNA isolation kit (Norgen Biotek Corp., Ontario, Canada). This DNA was submitted to the Center for Genomics and Bioinformatics at Indiana University for library preparation and Illumina NextSeq 150 (mid output) sequencing using paired-end reads. Next, bacteriophage genomes were assembled de novo using the sequencing analysis software, Geneious R10 (Biomatters, Auckland, New Zealand). To eliminate C. jejuni host sequences, all reads were mapped to the $C$. jejuni 81-176 reference genome (CP000538) and aligned reads were discarded from the dataset prior to assembly. Sequences were mapped using the Geneious Mapper function, with medium sensitivity, iterated up to 5 times. Next, the unused reads were assembled using the Geneious assembler with medium-low sensitivity. Then, the ORF finder function was used to identify putative ORFs, and protein sequences were analyzed via BLASTp to confirm assembled contigs were bacteriophage genomes

Identified phage genomes were clustered into phylogenetic clustering of the shared regions of these phages using VICTOR (43). VICTOR performs pairwise comparisons of phage nucleotide sequences using the (GBDP) approach (44) and the settings recommended for prokaryotic viruses (43). The resulting intergenomic distances (100 replicates each) were used to infer a balanced minimum evolution tree with branch support from FASTME and SPR post-processing for the D0 formula (31). 
The phylogenetic trees were rooted at the midpoint (45) and constructed by FigTree (46). Species, genus, and family level taxon boundaries were estimated by the OPTSIL program (33), recommended clustering thresholds (43), and an F value (fraction of links needed for cluster fusion) of 0.5 (47).

\subsection{Transmission electron microscopy}

To image phage isolates via TEM, a $10 \mu \mathrm{L}$ aliquot of phage suspension in SM buffer was transferred to a C-smart hydrophilic grid (Dune Sciences, Eugene, OR, USA). After $5 \mathrm{~min}$, the aliquot was removed from the grid via a lab tissue, followed by the addition of $1 \%$ uranyl acetate. The uranyl acetate stain was removed via a lab tissue after a 1 min exposure to the grid. The grids were then dried overnight at room temperature. Phage-loaded grids were inserted into a JEOL 2010F field-emission TEM (JEOL Ltd., Tokyo, Japan) and operated at $80 \mathrm{kV}$ for imaging.

\section{Acknowledgements}

Funding for this study was provided by a start-up fund from the University of Tennessee at Knoxville to JGJ. We acknowledge the University of Tennessee Genomics Core and the Center for Genomics and Bioinformatics at Indiana University for performing the sequencing in this study. We acknowledge Dali Qian of the Electron Microscopy Center at the University of Kentucky for TEM imaging. We also acknowledge Drs. lan and Pippa Connerton for their guidance regarding phage isolation methodology. 
Figures and Tables

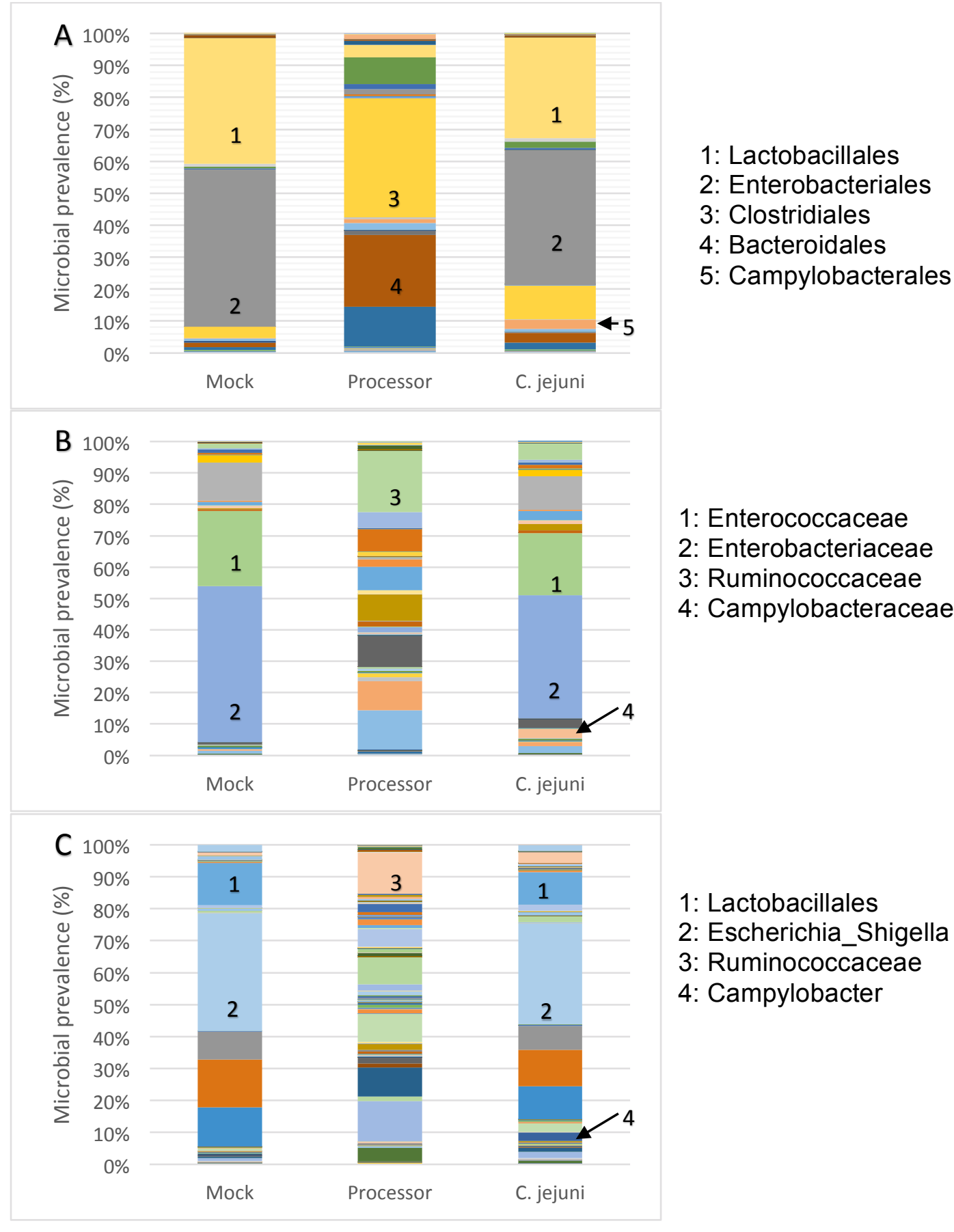

Figure 1. 16S rRNA OTU clustering of chicken ceca microbiomes. $(A)$ : Order; $(B)$ : Family; (C): Genus. Chickens were evaluated from a large-scale processor, inoculated at day-of-hatch with $C$. jejuni 81-176, or mock infected with PBS. Microbiome sequencing results was organized into OTUs determined via mothur (23). 

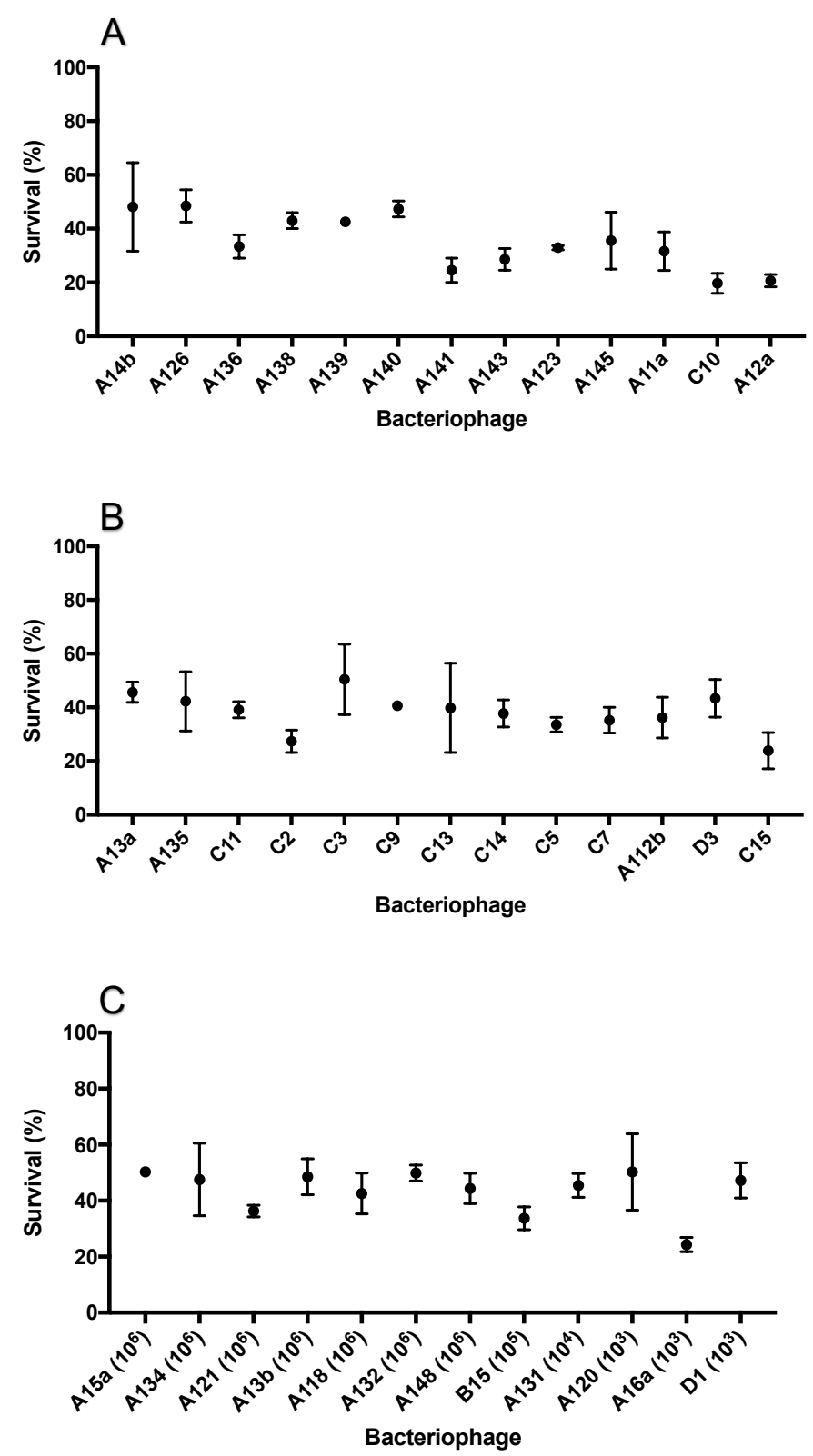

Figure 2. Percent growth of $C$. jejuni 81-176 at the bacteriophage titer (PFU) IC50 compared to a culture containing no phage. (A): 1 PFU; (B): $10^{1}$ PFU; (C): $10^{2}-10^{6}$ PFU. 


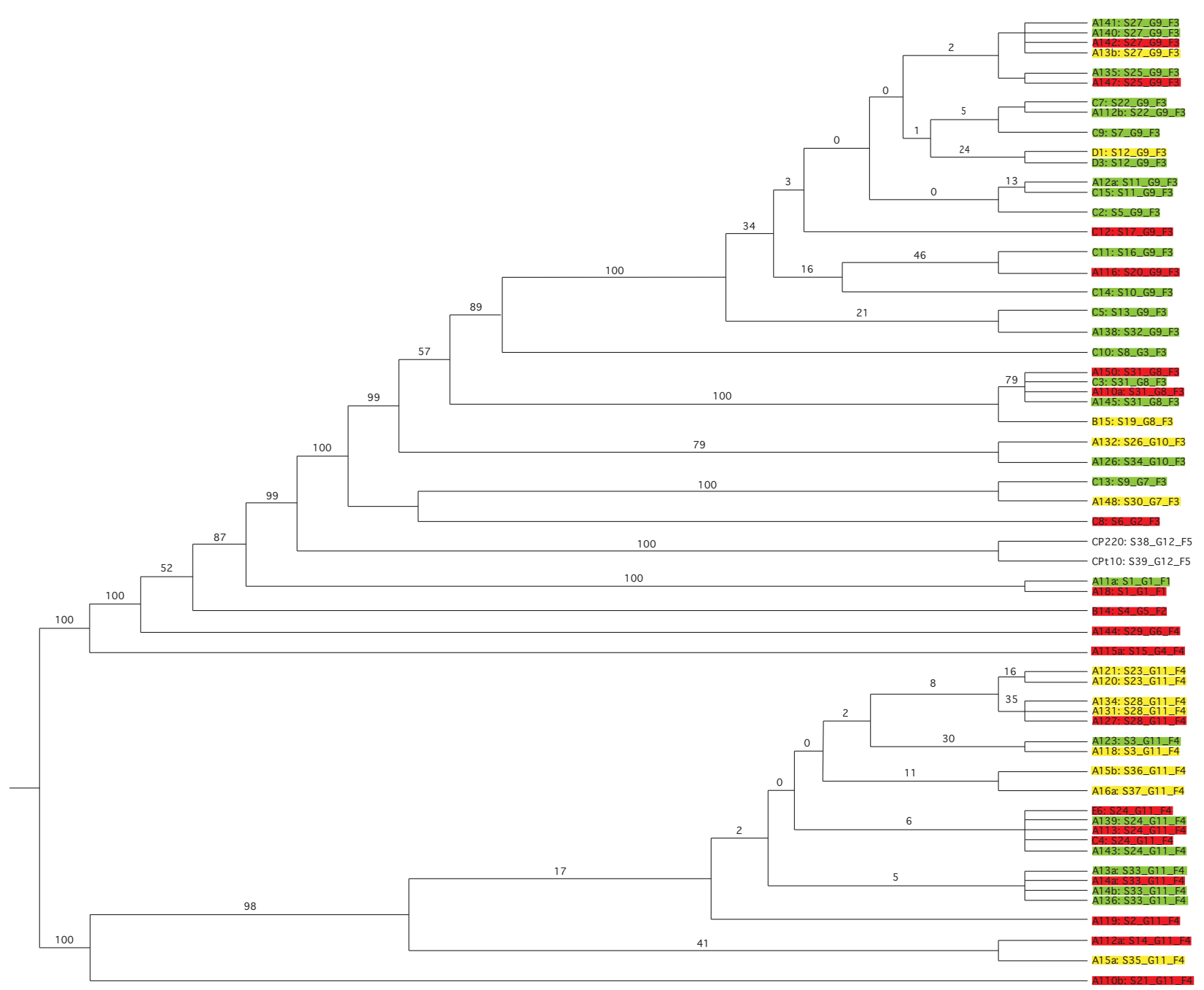

Figure 3. Bacteriophage diversity as determined by VICTOR (43). Phages highlighted green had an IC50 against $C$. jejuni $81-176$ at $1-10^{1} \mathrm{PFU}$. Phages highlighted yellow had an IC50 against $C$. jejuni at $10^{2}-10^{6}$ PFU. Phages highlighted red did not inhibit $C$. jejuni growth. The two phages not highlighted are described in a previous study (32). 


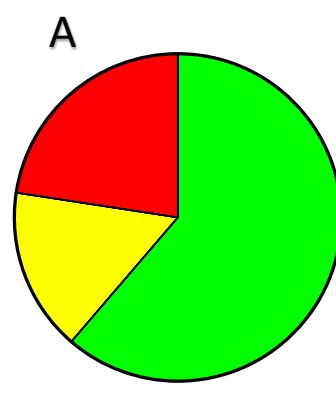

$$
\begin{array}{ll}
61.29 \% & 19 \\
16.13 \% & 5 \\
\hline 22.58 \% & 7
\end{array}
$$
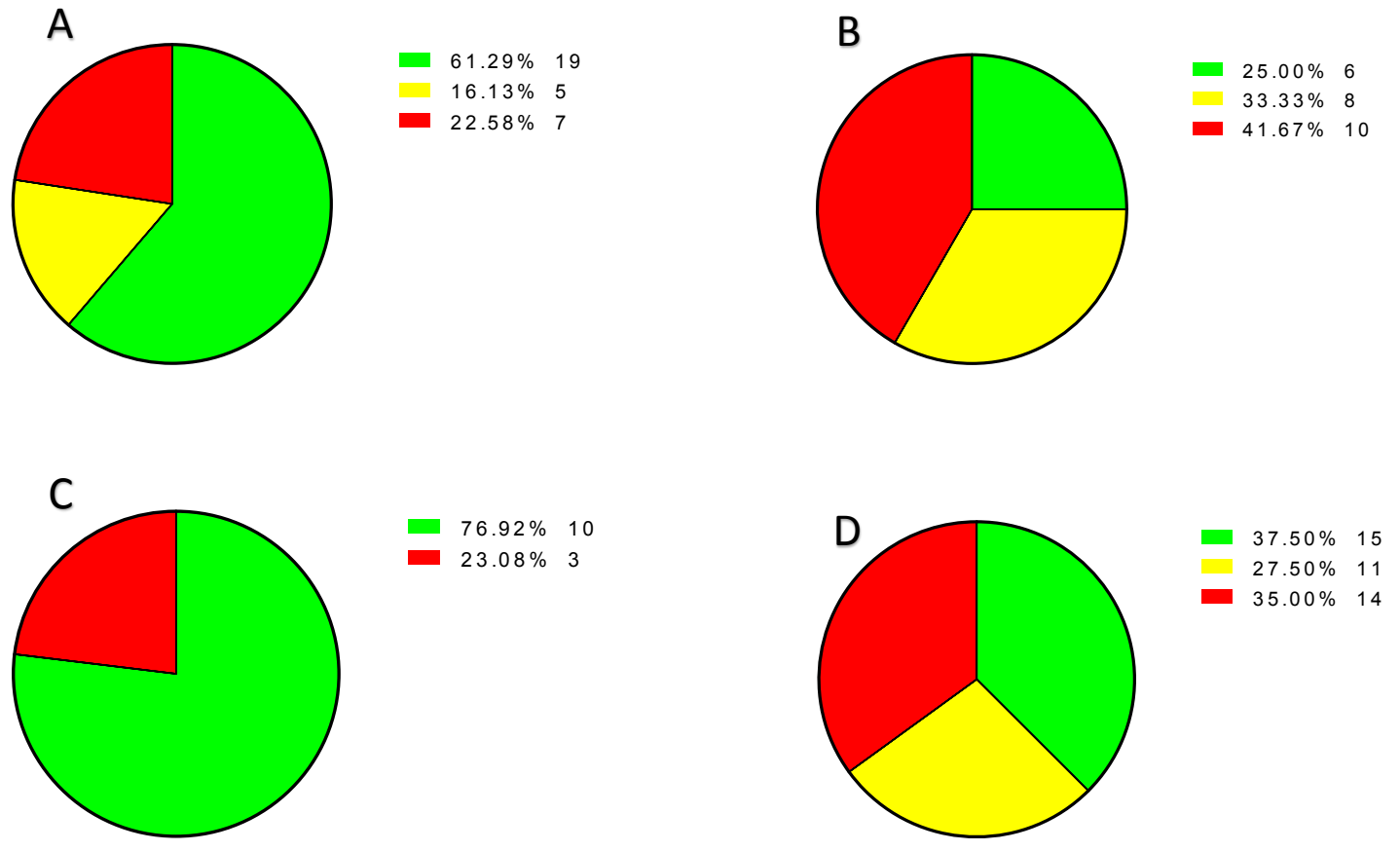

Figure 4. Distribution of phylogenomic clustering and isolation source of phage categorized by their ability to inhibit $C$. jejuni growth. (A): Clade 3; (B): Clade 4; (C): Untreated wastewater; (D): Chickens from a large processor. Chart legends show the percentage and raw value of each phage type. Green phages exhibited an IC50 against C. jejuni 81-176 at $1-10 \mathrm{PFU}$, yellow phages had an IC50 of $10^{2}-10^{6} \mathrm{PFU}$, red phages did not inhibit $C$. jejuni growth 


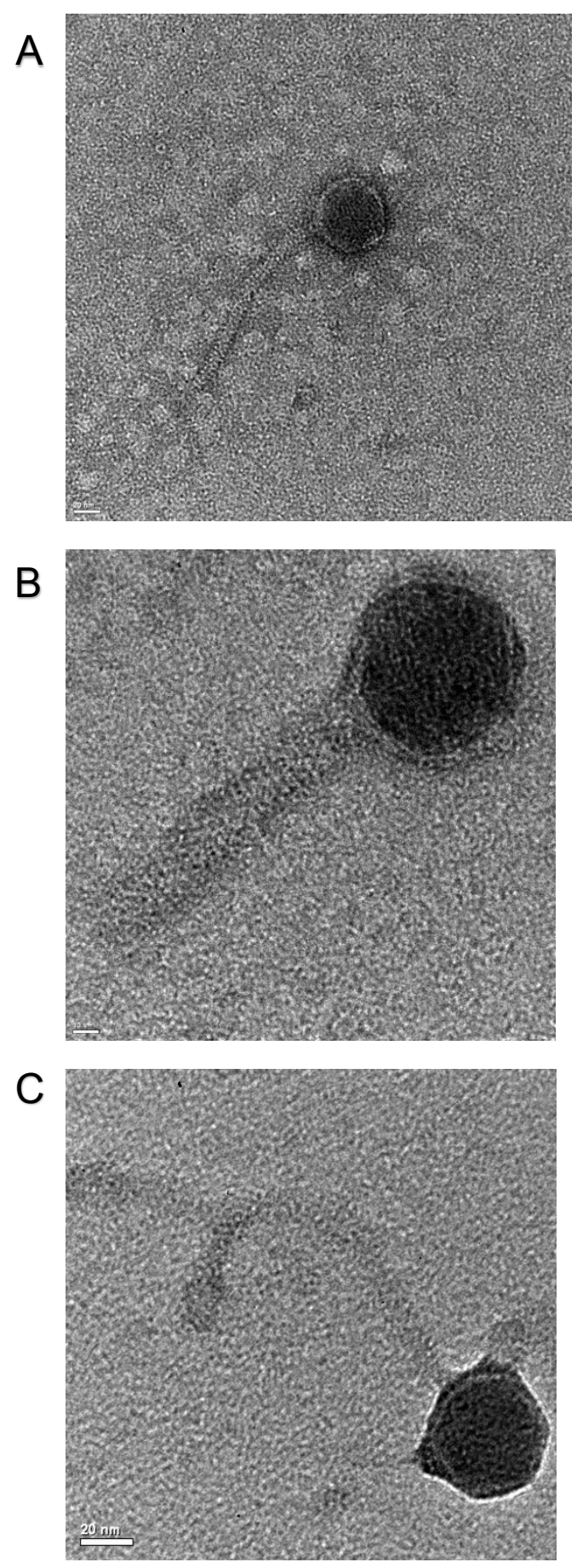

Figure 5: Transmission electron micrographs of bacteriophage. (A): Phage $\mathrm{C} 13$ (scale bar: $20 \mathrm{~nm}$ ); (B): Phage A126 (scale bar: $10 \mathrm{~nm}$ ); (C): Phage A136 (scale bar: 20 $\mathrm{nm})$. 
Table 1. Campylobacter counts from samples where bacteriophages were isolated. Each sample was a biological replicate.

\begin{tabular}{|l|l|}
\hline Environment & $\begin{array}{l}\text { Campylobacter } \\
\text { (CFU/g) }\end{array}$ \\
\hline Cow 1 & $5.6 \times 10^{5}$ \\
\hline Cow 2 & $9.7 \times 10^{4}$ \\
\hline Cow 3 & $3.0 \times 10^{3}$ \\
\hline Cow 4 & $2.7 \times 10^{6}$ \\
\hline Cow 5 & $1.4 \times 10^{4}$ \\
\hline Small Farm Chicken 1 & $2.7 \times 10^{6}$ \\
\hline Small Farm Chicken 2 & $3.2 \times 10^{7}$ \\
\hline Small Farm Chicken 3 & $7.9 \times 10^{6}$ \\
\hline Small Farm Chicken 4 & $5.9 \times 10^{6}$ \\
\hline Small Farm Chicken 5 & $2.0 \times 10^{6}$ \\
\hline Sheep 1 & $1.4 \times 10^{5}$ \\
\hline Sheep 2 & $2.8 \times 10^{5}$ \\
\hline Sheep 3 & $3.5 \times 10^{4}$ \\
\hline & \\
\hline
\end{tabular}

Table 2. Accession numbers for genome assemblies of Campylobacter-infecting bacteriophages.

\begin{tabular}{|l|l|}
\hline Bacteriophage & $\begin{array}{l}\text { Accession } \\
\text { Number }\end{array}$ \\
\hline A110a & MG065689 \\
\hline A110b & MG065688 \\
\hline A112a & MG065685 \\
\hline A112b & MG065682 \\
\hline A113 & MG065680 \\
\hline A115a & MG065678 \\
\hline A116 & MG065676 \\
\hline A118 & MG065675 \\
\hline A119 & MG065673 \\
\hline A11a & MG065691 \\
\hline A120 & MG065671 \\
\hline A121 & MG065670 \\
\hline A123 & MG065669 \\
\hline A126 & MG065668 \\
\hline A127 & MG065667 \\
\hline A12a & MG065666 \\
\hline A131 & MG065665 \\
\hline A132 & MG065664 \\
\hline A134 & MG065663 \\
\hline
\end{tabular}




\begin{tabular}{|c|c|}
\hline A135 & MG065690 \\
\hline A136 & MG065687 \\
\hline A138 & MG065684 \\
\hline A139 & MG065681 \\
\hline A13a & MG065679 \\
\hline A13b & MG065677 \\
\hline A140 & MG065674 \\
\hline A141 & MG065672 \\
\hline A142 & MG065662 \\
\hline A143 & MG065649 \\
\hline A144 & MG065648 \\
\hline A145 & MG065645 \\
\hline A147 & MG065644 \\
\hline A148 & MG065642 \\
\hline A14a & MG065641 \\
\hline A14b & MG065640 \\
\hline A150 & MG065639 \\
\hline A15a & MG065638 \\
\hline$A 15 b$ & MG065637 \\
\hline A16a & MG065636 \\
\hline A18a & MG065686 \\
\hline B14 & MG065683 \\
\hline B15 & MG065650 \\
\hline C10 & MG065651 \\
\hline C11 & MG065652 \\
\hline $\mathrm{C} 12$ & MG065635 \\
\hline C13 & MG065692 \\
\hline C14 & MG065653 \\
\hline C15 & MG065654 \\
\hline C2 & MG065655 \\
\hline $\mathrm{C} 3$ & MG065656 \\
\hline C4 & MG065657 \\
\hline C5 & MG065659 \\
\hline C7 & MG065658 \\
\hline $\mathrm{C} 8$ & MG065660 \\
\hline C9 & MG065661 \\
\hline D1 & MG065646 \\
\hline D3 & MG065647 \\
\hline E6 & MG065643 \\
\hline
\end{tabular}




\section{References}

1. Backert S, Boehm M, Wessler S, Tegtmeyer N. 2013. Transmigration route of Campylobacter jejuni across polarized intestinal epithelial cells: paracellular, transcellular or both? Cell Comm Signal 11:72.

2. Samuelson DR, Eucker TP, Bell JA, Dybas L, Mansfield LS, Konkel ME. 2013. The Campylobacter jejuni CiaD effector protein activates MAP kinase signaling pathways and is required for the development of disease. Cell Comm Signal 11:79.

3. Johnson TJ, Shank JM, Johnson JG. 2017. Current and potential treatments for reducing Campylobacter colonization in animal hosts and disease in humans. Front Microbiol 8.

4. Goldstein RER, Cruz-Cano R, Jiang C, Palmer A, Blythe D, Ryan P, Hogan B, White B, Dunn JR, Libby T. 2016. Association between community socioeconomic factors, animal feeding operations, and campylobacteriosis incidence rates: Foodborne Diseases Active Surveillance Network (FoodNet), 2004-2010. BMC Infect Dis 16:354.

5. Sharma VK, Johnson N, Cizmas L, McDonald TJ, Kim H. 2016. A review of the influence of treatment strategies on antibiotic resistant bacteria and antibiotic resistance genes. Chemosphere 150:702-714.

6. Hampton T. 2013. Report reveals scope of US antibiotic resistance threat. Jama 310:1661-1663.

7. Kumar A, Drozd M, Pina-Mimbela R, Xu X, Helmy YA, Antwi J, Fuchs JR, Nislow C, Templeton J, Blackall PJ. 2016. Novel anti-Campylobacter compounds identified using high throughput screening of a pre-selected enriched small molecules library. Front Microbiol 7.

8. El-Shibiny A, Scott A, Timms A, Metawea Y, Connerton P, Connerton I. 2009. Application of a group II Campylobacter bacteriophage to reduce strains of Campylobacter jejuni and Campylobacter coli colonizing broiler chickens. J Food Prot 72:733-740.

9. Zampara A, Sorensen MCH, Elsser-Gravesen A, Brondsted L. 2017. Significance of phagehost interactions for biocontrol of Campylobacter jejuni in food. Food Control 73: 11691175.

10. Atterbury RJ, Connerton PL, Dodd CE, Rees CE, Connerton IF. 2003. Isolation and characterization of Campylobacter bacteriophages from retail poultry. Appl Environ Microbiol 69: 4511-4518

11. El-Shibiny A, Connerton P, Connerton I. 2005. Enumeration and diversity of campylobacters and bacteriophages isolated during the rearing cycles of free-range and organic chickens. Appl Environl Microbiol 71:1259-1266.

12. Atterbury R, Dillon E, Swift C, Connerton P, Frost J, Dodd C, Rees C, Connerton I. 2005. Correlation of Campylobacter bacteriophage with reduced presence of hosts in broiler chicken ceca. Appl Environ Microbiol 71: 4885-4887.

13. Brüssow H, Mcgrath S, Van Sinderen D. 2007. Phage therapy: the Western perspective. Norfolk, UK: Caister Acad. Press.

14. Connerton P, Connerton I, Barrow P, Seal B, Atterbury R, Nachamkin I. 2006. Campylobacter and their bacteriophage in poultry. Avian gut function in health and disease: 311-321. 
15. Brüssow H. 2012. What is needed for phage therapy to become a reality in Western medicine? Virology 434:138-142.

16. Grajewski B, Kusek J, Gelfand H. 1985. Development of a bacteriophage typing system for Campylobacter jejuni and Campylobacter coli. J Clin Microbiol 22:13-18.

17. Hansen VM, Rosenquist H, Baggesen DL, Brown S, Christensen BB. 2007. Characterization of Campylobacter phages including analysis of host range by selected Campylobacter Penner serotypes. BMC Microbiol 7:90.

18. Carrillo CML, Connerton PL, Pearson T, Connerton IF. 2007. Free-range layer chickens as a source of Campylobacter bacteriophage. Antonie Van Leeuwenhoek 92:275.

19. Salama S, Bolton F, Hutchinson D. 1989. Improved method for the isolation of Campylobacter jejuni and Campylobacter coli bacteriophages. Lett Appl Microbiol 8:5-7.

20. Pearson A, Greenwood M, Healing T, Rollins D, Shahamat M, Donaldson J, Colwell R. 1993. Colonization of broiler chickens by waterborne Campylobacter jejuni. Appl Environl Microbiol 59:987-996.

21. Newell D, Fearnley C. 2003. Sources of Campylobacter colonization in broiler chickens. Appl Environ Microbiol 69:4343-4351.

22. Stern N, Fedorka-Cray P, Bailey J, Cox N, Craven S, Hiett K, Musgrove M, Ladely S, Cosby D, Mead G. 2001. Distribution of Campylobacter spp. in selected US poultry production and processing operations. J Food Prot 64:1705-1710.

23. Kozich JJ, Westcott SL, Baxter NT, Highlander SK, Schloss PD. 2013. Development of a dual-index sequencing strategy and curation pipeline for analyzing amplicon sequence data on the MiSeq Illumina sequencing platform. Appl Environ Microbiol 79:5112-5120.

24. Wei S, Morrison M, Yu Z. 2013. Bacterial census of poultry intestinal microbiome. Poult Sci 92:671-683.

25. Sergeant MJ, Constantinidou C, Cogan TA, Bedford MR, Penn CW, Pallen MJ. 2014. Extensive microbial and functional diversity within the chicken cecal microbiome. PLoS One 9:e91941.

26. Qu A, Brulc JM, Wilson MK, Law BF, Theoret JR, Joens LA, Konkel ME, Angly F, Dinsdale EA, Edwards RA. 2008. Comparative metagenomics reveals host specific metavirulomes and horizontal gene transfer elements in the chicken cecum microbiome. PLoS One

27. Hyman P, Abedon ST. 2010. Bacteriophage host range and bacterial resistance. Adv Appl Microbiol 70:217-248.

28. Sails A, Wareing D, Bolton F, Fox A, Curry A. 1998. Characterisation of 16 Campylobacter jejuni and $C$. coli typing bacteriophages. J Med Microbiol 47:123-128.

29. Coward C, Grant AJ, Swift C, Philp J, Towler R, Heydarian M, Frost JA, Maskell DJ. 2006. Phase-variable surface structures are required for infection of Campylobacter jejuni by bacteriophages. Appl Environ Microbiol 72:4638-4647.

30. Aidley J, Sorensen MCH, Bayliss CD, Bronsted L. 2017. Phage exposure causes dynamic shifts in the expression states of specific phase-variable genes of Campylobacter jejuni. Microbiology 163: 911-919.

31. Lefort V, Desper R, Gascuel O. 2015. FastME 2.0: a comprehensive, accurate, and fast distance-based phylogeny inference program. Mol Biol Evol 32:2798-2800. 
32. Timms AR, Cambray-Young J, Scott AE, Petty NK, Connerton PL, Clarke L, Seeger K, Quail $\mathrm{M}$, Cummings N, Maskell DJ. 2010. Evidence for a lineage of virulent bacteriophages that target Campylobacter. BMC Genomics 11:214.

33. Göker M, García-Blázquez G, Voglmayr H, Tellería MT, Martín MP. 2009. Molecular taxonomy of phytopathogenic fungi: a case study in Peronospora. PLoS One 4:e6319.

34. Javed MA, Ackermann H-W, Azeredo J, Carvalho CM, Connerton I, Evoy S, Hammerl JA, Hertwig S, Lavigne R, Singh A. 2014. A suggested classification for two groups of Campylobacter myoviruses. Arch Virol 159:181-190.

35. Carrillo CL, Atterbury R, El-Shibiny A, Connerton P, Dillon E, Scott A, Connerton I. 2005. Bacteriophage therapy to reduce Campylobacter jejuni colonization of broiler chickens. Appl Environ Microbiol 71:6554-6563.

36. Nagel TE, Chan BK, De Vos D, El-Shibiny A, Kang'ethe EK, Makumi A, Pirnay J-P. 2016. The developing world urgently needs phages to combat pathogenic bacteria. Front Microbiol 7.

37. Amour C, Gratz J, Mduma E, Svensen E, Rogawski ET, McGrath M, Seidman JC, McCormick BJ, Shrestha S, Samie A. 2016. Epidemiology and impact of Campylobacter infection in children in 8 low-resource settings: Result from the MAL-ED study. Clin Infect Dis 63:1171-1179.

38. Korlath JA, Osterholm MT, Judy LA, Forfang JC, Robinson RA. 1985. A point-source outbreak of campylobacteriosis associated with consumption of raw milk. J Infect Dis 152:592-596.

39. Black RE, Levine MM, Clements ML, Hughes TP, Blaser MJ. 1988. Experimental Campylobacter jejuni infection in humans. J Infect Dis 157:472-479.

40. Richardson JW, Schumann K, Feldman P. 2006. Simulation for applied risk management. Department of Agricultural Economics, Agricultural and Food Policy Center, Texas A\&M University.

41. Johnson JG, Livny J, DiRita VJ. 2014. High-throughput sequencing of Campylobacter jejuni insertion mutant libraries reveals mapA as a fitness factor for chicken colonization. J Bacteriol 196:1958-1967.

42. Illumina. 2017. 16S metagenomic sequencing library preparation.

43. Meier-Kolthoff JP, Goeker M. 2017. VICTOR: Genome-based Phylogeny and Classification of Prokaryotic Viruses. bioRxiv:107862.

44. Meier-Kolthoff JP, Auch AF, Klenk H-P, Göker M. 2013. Genome sequence-based species delimitation with confidence intervals and improved distance functions. BMC Bioinformatics 14:60.

45. Farris JS. 1972. Estimating phylogenetic trees from distance matrices. Am Nat 106:645668.

46. Rambaut A. 2006. A graphical viewer of phylogenetic trees and a program for producing publication-ready figures. http://tree.bio.ed.ac.uk/software/figtree/.

47. Meier-Kolthoff JP, Hahnke RL, Petersen J, Scheuner C, Michael V, Fiebig A, Rohde C, Rohde M, Fartmann B, Goodwin LA. 2014. Complete genome sequence of DSM 30083 T, the type strain (U5/41 T) of Escherichia coli, and a proposal for delineating subspecies in microbial taxonomy. Stand Genomic Sci 9:2. 